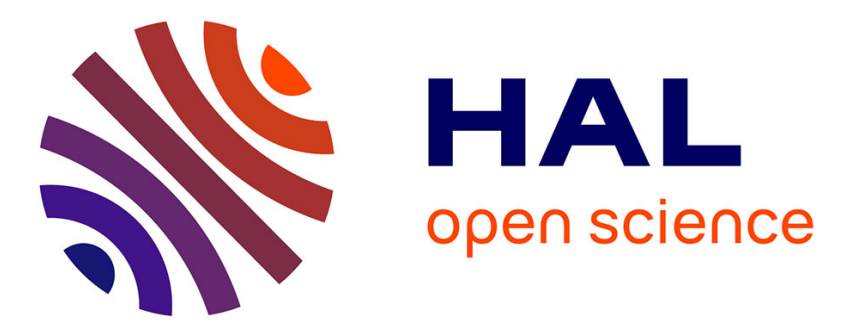

\title{
Une pédagogie du droit sous contrainte. Les syndicalistes et les inspecteurs du travail dans l'activité de consultation juridique
}

\author{
Laurent Willemez
}

\section{- To cite this version:}

Laurent Willemez. Une pédagogie du droit sous contrainte. Les syndicalistes et les inspecteurs du travail dans l'activité de consultation juridique. Politix, 2017, Justice au travail, 118, pp.103-130. 10.3917/pox.118.0103 . halshs-01616740

\section{HAL Id: halshs-01616740 \\ https://shs.hal.science/halshs-01616740}

Submitted on 27 Oct 2017

HAL is a multi-disciplinary open access archive for the deposit and dissemination of scientific research documents, whether they are published or not. The documents may come from teaching and research institutions in France or abroad, or from public or private research centers.
L'archive ouverte pluridisciplinaire HAL, est destinée au dépôt et à la diffusion de documents scientifiques de niveau recherche, publiés ou non, émanant des établissements d'enseignement et de recherche français ou étrangers, des laboratoires publics ou privés. 


\section{UNE PÉDAGOGIE DU DROIT SOUS CONTRAINTE. LES SYNDICALISTES ET LES INSPECTEURS DU TRAVAIL DANS L'ACTIVITÉ DE CONSULTATION JURIDIQUE} Laurent Willemez

De Boeck Supérieur | « Politix »

2017/2 n 118 | pages 103 à 130

ISSN 0295-2319

ISBN 9782807391246

Article disponible en ligne à l'adresse :

http://www.cairn.info/revue-politix-2017-2-page-103.htm

\section{Pour citer cet article :}

Laurent Willemez, «Une pédagogie du droit sous contrainte. Les syndicalistes et les inspecteurs du travail dans l'activité de consultation juridique », Politix 2017/2 $\left(\mathrm{n}^{\circ} 118\right), \mathrm{p} .103-130$.

DOI 10.3917/pox.118.0103

Distribution électronique Cairn.info pour De Boeck Supérieur.

(C) De Boeck Supérieur. Tous droits réservés pour tous pays.

La reproduction ou représentation de cet article, notamment par photocopie, n'est autorisée que dans les limites des conditions générales d'utilisation du site ou, le cas échéant, des conditions générales de la licence souscrite par votre établissement. Toute autre reproduction ou représentation, en tout ou partie, sous quelque forme et de quelque manière que ce soit, est interdite sauf accord préalable et écrit de l'éditeur, en dehors des cas prévus par la législation en vigueur en France. Il est précisé que son stockage dans une base de données est également interdit. 


\title{
Une pédagogie du droit sous contrainte. Les syndicalistes et les inspecteurs du travail dans l'activité de consultation juridique
}

\author{
Laurent WILLEMEZ
}

\begin{abstract}
Résumé - L'objectif de l'article est d'étudier une activité juridique rarement prise en compte dans les travaux sur les usages syndicaux du droit du travail : les consultations juridiques au niveau local. L'analyse localisée des consultations juridiques dans des espaces différents où sont donnés des conseils juridiques aux salariés que sont les unions locales de trois syndicats (CGT, CFDT et FO) et la direction départementale du travail montre la spécificité de ces conseils : ils constituent d'abord un travail de filtrage de la demande des salariés, souvent démunis, et une première forme de traduction juridique des sentiments d'injustice et des difficultés vécues par les salariés dans leur entreprise. Dès lors, les acteurs chargés de ces conseils, militants syndicaux et agents de contrôle de l'inspection du travail, considèrent globalement ce travail comme une activité politique de défense des salariés dans des relations de travail asymétriques. L'article insiste ensuite sur les différences entre les deux groupes de "fournisseurs " de conseil, en montrant comment l'exercice de ces activités est lié à des logiques organisationnelles et d'ethos professionnel.
\end{abstract}


vertissement, sentiment d'imminence d'une procédure de licenciement,
baisse de salaires, modification des heures de travail, crainte pour sa
santé, sentiment de harcèlement... Nombre de salariés vivent ces difficultés au travail comme autant de craintes pour leur emploi, et elles nourrissent un sentiment d'injustice qui doit être exprimé, et éventuellement réparé. Face à ces vécus douloureux, ces travailleurs peuvent chercher à se protéger des risques sociaux que sont le chômage ou la maladie, à mettre des mots derrière le malaise et retrouver une partie de la dignité perdue, ou encore à " obtenir justice et réciprocité ${ }^{1}$ » de la part de leur employeur ou de leurs managers. Mais à qui doivent-ils s'adresser pour ce faire ? Dans une entreprise, un établissement de grande taille ou une structure qui fait l'objet d'une implantation syndicale forte, les institutions de représentation du personnel jouent ce rôle d'interlocuteur. Pour les salariés qui disposent de ressources sociales et juridiques, en particulier pour les cadres, le cabinet d'avocat constitue une autre solution possible. Mais dans les PME ou les TPE, et plus largement pour ces travailleurs sinon isolés du moins éloignés de la protection et des possibilités d'actions des syndicats et des représentants des salariés, il existe deux espaces, "lieux d'écoute ${ }^{2}$ » mais aussi lieux de production de solutions : les consultations juridiques des organisations syndicales et les consultations de l'inspection du travail.

L'observation ethnographique de ces consultations, couplée à des entretiens avec les agents qui en sont chargés, militants syndicaux spécialisés dans le droit du travail d'une part, agents de contrôle de l'administration du travail (en particulier contrôleurs du travail) d'autre part, donne ainsi à voir des acteurs à l'écoute de salariés exprimant ces difficultés et ces sentiments d'injustice, démêlant les tenants et les aboutissants par leurs questions et tentant de percevoir en quoi le droit du travail tel qu'il s'exprime dans le Code du travail, dans les conventions collectives, dans le règlement intérieur de l'entreprise ou dans le contrat de travail, peut ou non permettre d'agir sur la situation, de rétablir des droits ou de faire justice aux demandeurs. Par un travail d'écoute, de diagnostic et finalement de traduction en catégories juridiques, ils font des consultations juridiques des lieux d'usage, et par conséquent de production, du droit du travail. Ces espaces sont donc des lieux susceptibles d'être étudiés à l'aide de ce que l'on appelle la « sociologie du guichet », par l'analyse des agents, militants ou professionnels, qui entrent en contact avec le public, de la manière dont ils construisent leur rôle, ainsi que des catégories de classements et des jugements moraux qu'ils produisent à l'occasion de ces interactions.

1. Pour traduire de manière approximative le très beau titre du livre de Sally Engle Merry: Getting Justice and Getting Even: Legal Consciousness Among Working-Class Americans, Chicago, University of Chicago Press, 1990.

2. Fassin (D.), Des maux indicibles. Sociologie des lieux d'écoute, Paris, La Découverte, 2004. 
Mais ces « guichets » ont ici une particularité, qui est celle de manipuler des catégories et des textes juridiques. Les particularités du champ juridique se diffractent ainsi sur les différents acteurs de l'interaction. D'abord parce que sont mis aux prises des agents ne possédant pas, de part et d'autre de la table où se tient l'interaction, les mêmes rapports à ce que nous avons pu appeler des dispositions juridiques ${ }^{3}$. Tout particulièrement, l'interaction fait se rencontrer des spécialistes du droit et des profanes, souvent fortement dominés ou ne disposant que rarement de ressources leur permettant de s'y retrouver et de jouer avec le droit. Si ces derniers ne possèdent le plus souvent pas de conscience théorique des enjeux et des potentialités du droit, on peut néanmoins montrer qu'ils détiennent une sorte de sens pratique du droit qui leur est applicable, et qui les autorise d'ailleurs à croire qu'ils sont dans leur droit. C'est sans doute dans cette perspective qu'on peut parler, pour les travailleurs les plus dénués de capital culturel et de ressources juridiques, de " conscience du droit », en reprenant par là les chercheurs états-uniens travaillant sur la production ordinaire de la légalité 4 . Par rapport à cette conscience, le rôle des agents de consultation peut alors être de les conforter dans leur croyance ou au contraire de les rappeler à l'ordre juridique. Travail de filtrage, de traduction, la consultation apparaît ainsi comme une activité de pédagogie du droit et de socialisation au juridique.

Les deux types d'agents de consultation partagent donc une position commune. En cela, ils peuvent être qualifiés d'«intermédiaires du droit»: acteurs juridiques même s'ils ne peuvent pas véritablement être qualifiés de professionnels du droit au sens où ils n'appartiennent pas à un corps considéré comme occupant une position centrale dans le champ juridique (même si, comme on le verra, la question est compliquée pour les inspecteurs du travail dont l'activité est juridique de part en part), ils se donnent pour rôle de diffuser le droit du travail et de montrer aux salariés la manière dont celui-ci peut ou non contribuer à les protéger, à leur rendre justice, à les défendre en cas de manquement de l'employeur ou du manager. Comme on le montrera, ils partagent aussi le plus souvent une connaissance importante du droit du travail dans son aspect le plus individualiste, c'est-à-dire lié au contrat de travail, son exécution, ses conditions d'effectuation et sa rupture. Et comme pour tous les détenteurs de cette disposition juridique, ils possèdent aussi une même croyance dans le droit comme mode monopolistique de régulation et de résolution des conflits au sein du monde du travail. L'objectif est donc bien de montrer comment un certain nombre d'acteurs, à travers leur rôle d'intermédiaires du droit - au sens que Jérôme Pélisse donne à ce terme ${ }^{5}-$, produisent des formes de conscience du droit.

3. Willemez (L.), «Un champ mis à l'épreuve. Structure et propriétés du champ juridique dans la France contemporaine ", Droit et société, 89, 2015.

4. Ewick (P.), The Common Place of Law: Stories from Everyday Life, Chicago, University of Chicago Press, 1998 ; Pélisse (J.), «A-t-on conscience du droit ? Autour des Legal Consciousness Studies », Genèses, 59, 2005. 5. Pélisse (J.), Le travail du droit. Trois enquêtes sur la légalité ordinaire, mémoire pour l'habilitation à diriger des recherches, IEP de Paris, 2014. 
Mais réunir ces acteurs sous l'identité commune d' « intermédiaires du droit » ne doit pas masquer leurs nombreuses différences. En termes de sociologie des groupes professionnels, on montrera dans quelle mesure on se retrouve face à deux groupes qui ont des propriétés très différentes, les conduisant à des pratiques hétérogènes en termes de consultations juridiques. L'activité juridique des militants syndicaux est désormais bien documentée, et l'on sait dans quelle mesure leur position à mi-chemin du champ juridique et de l'activité militante les fragilise en même temps qu'elle les renforce : exerçant une activité spécialisée, ils occupent ce faisant une position spécifique au sein de leur organisation, faite de distance mais aussi parfois de suspicion de la part des autres militants. Dans le même temps, leur façon de travailler avec le droit du travail, en cherchant à lui donner un «sens » syndical et en s'efforçant de le faire entrer dans des stratégies plus larges, en fait des intermédiaires du droit spécifiques. De leur côté, les inspecteurs sont des agents de l'État, et c'est leur rapport à la neutralité, la manière dont ils articulent les injonctions souvent contradictoires dans lesquelles ils sont pris qui guident leur activité de consultation. L'homogénéité de chacun ces groupes n'est d'ailleurs qu'assez relative : du côté de l'inspection du travail, les acteurs des consultations sont des contrôleurs du travail, agents de catégorie B, et sur le terrain d'observation on n'a pas noté de différence dans leur conception du métier. Du côté des syndicalistes, l'homogénéité est moins forte du fait des appartenances syndicales diverses, mais on retrouve tout de même une manière commune de pratiquer l'activité de conseil aux salariés.

\section{Encadré 1. Méthode d'enquête}

La recherche sur laquelle s'appuie cet article avait pour objet de comparer les consultations juridiques pour trois groupes d'acteurs intervenant dans le droit du travail et spécialisés dans la défense des salariés : syndicalistes, inspecteurs du travail et avocats (à l'enquête ethnographique s'ajoutait une étude exhaustive de l'encadrement juridique du travail de consultation) dans deux départements français ('’un dans l'Ouest de la France, l'autre en région parisienne) ${ }^{6}$. Pour l'article, seules les données concernant le département de l'Ouest de la France ont été mobilisées, principalement de manière à réfléchir aux logiques locales de l'engagement syndical. Le lieu d'étude principal a été le chef-lieu du département, qui est aussi une capitale régionale. Des observations ont été réalisées sur des demi-journées de consultation : deux séances pour les agents de contrôle, trois séances pour les militants syndicaux. Pour les observations avec les agents de contrôleurs, nous étions en retrait de l'interaction, assis à une table un peu plus loin; concernant les syndicalistes, l'enquêteur et l'enquêtrice étaient assis à côté d'eux et étaient présentés comme des personnes venant étudier le fonctionnement d'une consultation juridique. L'accueil

6. La recherche a été financée par la mission de recherche Droit et justice (convention de recherche $\mathrm{n}^{\circ}$ 212.01.18.16). Elle a donné lieu à un rapport de recherche : Bonnin (V.), Willemez (L.), Accès au droit et défense des salariés: entre service et apostolat, Paris, Mission de recherche Droit et justice, 2015. 
a toujours été très positif. Des entretiens ont été réalisés ensuite avec les acteurs des consultations, ainsi qu'avec les différents responsables locaux des syndicats, ainsi que le directeur départemental adjoint du travail ${ }^{7}$. Lors des entretiens, nous leur demandions de revenir sur les cas que nous avions observés. Il ne nous a pas été possible de nous entretenir avec les bénéficiaires des consultations, dont nous n'avons donc que les informations qui transparaissaient des récits réalisés pendant les séances de consultation.

\section{Entre traduction et pédagogie du droit : les consultations juridiques des organisations syndicales comme activité militante}

\section{Les dispositifs de consultation}

Les dispositifs de permanences juridiques sont principalement marqués par leur extrême hétérogénéité selon les départements et les organisations syndicales. Il est d'ailleurs fort difficile d'indexer les différences entre les unions départementales et les rapports au droit exprimés et revendiqués au niveau national. Dans ce domaine comme dans beaucoup d'autres, on retrouve une extraordinaire diversité des situations selon les territoires ${ }^{8}$. L'analyse au niveau d'un département permet de montrer que les caractéristiques de ces permanences et leur nombre renvoient à des rapports au droit spécifiques selon les organisations mais aussi à une sorte de division du travail entre organisations.

Ainsi dans le département étudié, les permanences juridiques sont principalement le fait de la CGT, de la CFDT et de Force ouvrière (FO), cette dernière ayant pris une position dominante dans ce secteur de l'activité juridique, principalement du fait de la présence d'un responsable syndical qui a investi toutes ses forces militantes dans l'action juridique, en particulier prud'homale. Si ce militant a quitté l'organisation pour fonder une sorte de permanence privée dans le chef-lieu du département, cette idiosyncrasie locale et syndicale explique une forte présence du syndicat dans le secteur de la consultation juridique. La CGT du département s'est pour sa part conformée formellement à la position méfiante de la confédération envers le conseil juridique, puisqu'un congrès de l'union départementale de ce syndicat a décidé, en 2006, de mandater systématiquement des avocats concernant les questions juridiques ; par conséquent, les permanences ne se veulent plus directement juridiques mais beaucoup plus

\footnotetext{
7. Une partie du travail de terrain a été réalisée par Angelina Garrin, ancienne doctorante du GRESCO.

8. Sur ce point, il faut noter qu'un certain nombre de travaux récents se sont centrés sur les usages du droit dans les organisations syndicales. Pour autant, à notre connaissance, il n'existe aucune étude sur les configurations locales mettant aux prises activité juridique et activité syndicale (sinon éventuellement au niveau de l'entreprise). Cf. Narritsens (A.), Pigenet (M.), dir., Pratiques syndicales du droit. France XX $X^{e}-X X I^{e}$ siècles, Rennes, Presses universitaires de Rennes, 2014 ; Béroud (S.), Denis (J.-M.), Desage (F.), Pélisse (J.), La lutte continue? Les conflits du travail dans la France contemporaine, Bellecombe-en-Bauges, Éditions du Croquant, 2008.
} 
larges. Pour autant, il semble que malgré cette décision, et par la demande même du public venu demander conseil, la plus grande partie des demandes renvoient à des questions de droit liées au contrat de travail. Il en est de même à l'union départementale de la CFDT, pour laquelle les consultations sont " polyvalentes, [même si] elles sont, quand même, un peu repérées comme un lieu d'accès à des renseignements d'ordre juridique », comme le dit le secrétaire d'une union locale. Il faut dire qu'au niveau national, la CFDT a un positionnement distant et peu confiant envers les activités juridiques de leurs permanents, même si celles-ci entrent dans l'effort de syndicalisation. Ces rapports compliqués aux permanences juridiques renvoient d'abord aux positions nationales des deux syndicats face au juridique, qui seraient susceptibles de mettre de côté le caractère collectif, voire agonistique pour la CGT, de l'activité syndicale. Et dans le même temps, la demande de droit des salariés est tellement forte que, comme si une sorte de loi d'airain du juridisme était suivie, et ce malgré les positions politiques les plus volontaristes, les permanences redeviennent rapidement des lieux où il est d'abord question de droit du travail.

C’est donc FO qui a, dans le département, l'activité juridique la plus importante, la plus institutionnalisée et la plus organisée, ce qui renvoie à l'intérêt de la confédération pour ce type d'activité syndicale, qui lui permet de donner force et même parfois vie au niveau interprofessionnel. Les responsables de l'union départementale ont toujours présenté les consultations juridiques comme l'un de leurs fleurons. Organisée pour la première fois en 1992, la permanence juridique a un grand succès; elle est quotidienne, les militants se relayant au cours de la semaine. Le « service juridique », puisque c'est ainsi qu'il est dénommé, est situé dans un local spécifique, tout proche d'un service de défense des consommateurs. On pourrait évoquer une véritable professionnalisation du rôle, si les militants n'étaient pas tous des retraités. Le site Internet est d'ailleurs très clair sur l'importance donnée au juridique :

«Vous êtes seul face à une question, une situation difficile ? Respect du droit du travail et de la convention collective, pressions psychologiques et harcèlement, sanctions disciplinaires et licenciement, élections professionnelles et instances élues du personnel, reconnaissance des compétences et respect des rémunérations, échanges de correspondances avec l'employeur, entretien préalable au licenciement... Conseils, stratégies, accompagnement juridique... Je vous écoute ! »

Cela témoigne de la place centrale occupée par le juridique à Force ouvrière, dans la mesure où cette activité participe à l'image de marque de l'organisation au niveau local, comme l'explique le secrétaire général de l'union départementale au moment de l'enquête :

«On veut vraiment faire en sorte que l'image de l'organisation pour les salariés et pour les institutions extérieures soit la meilleure possible. On y met donc les moyens. Donc en fait chaque mois, on a un planning avec le nom des personnes et les horaires de leur présence. [Il sort d'une chemise cartonnée 
le planning des permanences juridiques FO pour le mois de décembre et me le donne.] [...]. En sachant que moi, je suis là en permanence. Mon secrétariat est là en permanence. On a des permanents syndicaux qui viennent, qui passent et qui sont amenés ponctuellement à combler les zones blanches. Les personnes qui tiennent les permanences juridiques sont très souvent retraitées, et bien souvent de très jeunes retraités. »

\section{Une activité " proto-juridique » ?}

Au-delà des spécificités stratégiques et organisationnelles, le travail de consultation juridique prend souvent la même forme, celle de l'écoute du récit, souvent profus voire confus, parfois nourri d'émotion, que fait le salarié. La lecture des documents apportés par les salariés (bulletin de paie, contrat de travail, lettre d'avertissement...) ponctue l'écoute, l'ensemble permettant au militant de dresser un diagnostic juridique. Comme le montre Aaron Cicourel pour les médecins, les consultations sont l'occasion de produire une " abstraction étayée sur un contenu », mais aussi une «abstraction par recodage», le tout permettant de traduire des savoirs profanes en discours formalisés, ou au moins un discours permettant de confronter la réalité du récit aux catégories juridiques du Code du travail ou de la convention collective ${ }^{9}$. Ce recodage permet alors, par un effet de boucle, de proposer des solutions concrètes, qui en réalité prennent souvent la forme d'une lettre rédigée à l'attention de l'employeur. L'encadré 2 restitue longuement une de ces consultations, montrant ces allers-retours entre construction du récit, traduction en catégories juridiques plus ou moins formelles et conseils concrets.

\section{Encadré 2. Une consultation juridique auprès d'une syndicaliste}

Mauricette reçoit une salariée d'une vingtaine d'années, accompagnée de son père. La jeune femme, en contrat d'insertion en tant que travailleuse handicapée (elle semble avoir un handicap mental léger), qui a un fils de cinq ans, travaille depuis un an dans un restaurant. Elle rencontre des difficultés à défendre ses congés et ses arrêts de travail. Plus largement, il semble que le gérant du restaurant souhaite se débarrasser d'elle pour des raisons que nous ne connaîtrons pas. La jeune femme a beaucoup de difficultés à faire le récit de ce qui lui arrive, si bien qu'elle est parfois aidée par son père. À plusieurs reprises au cours de l'entretien, Mauricette lui demande si elle a des photocopies de ses arrêts de travail ou d'autres documents nécessaires pour comprendre la situation. Ce n'est jamais le cas. Mauricette lui explique à quel point ces papiers sont importants, qu'il faut les chercher, qu'elle ne peut se contenter de noter des dates approximatives sans les documents qui en attesteraient.

À un moment de l'entretien, Mauricette explique à la salariée et à son père qu'il faut consulter la convention collective. «Quelle est votre convention? » La jeune femme

9. Cicourel (A. V.), Le raisonnement médical. Une approche socio-cognitive, Paris, Seuil, 2002, p. 84-85. 
semble ne pas comprendre la demande. La syndicaliste explique ensuite assez longuement les règles juridiques concernant les congés payés et dans quelle mesure le fait qu'elle ait un enfant change la donne. La salariée écoute sans rien dire. Le père reprend alors la parole et explique que sa fille est déjà allée voir l'inspection du travail. On lui a expliqué que celle-ci devait être avisée de ses congés un mois avant, ce que l'employeur ne fait pas. Elle est ensuite allée voir directement la gérante pour en parler avec elle mais cette dernière lui a dit de lui envoyer des recommandés. Mauricette dit: «Ce n'est pas plus mal! C’est même la meilleure solution!» Il restera ainsi des traces de ses demandes.

Face aux questions de Mauricette, la jeune femme a du mal à s'exprimer et s'y reprend à plusieurs fois pour expliquer sa situation. Ce qu'elle raconte est souvent confus. Pendant son récit, Mauricette lui pose des questions concrètes et lui demande des exemples. La salariée explique notamment que la gérante fait tout pour l'« emmerder » en lui refusant des vacances pendant l'été, en ne la prévenant pas à l'avance de ses congés ou en lui donnant des vacances à certaines dates alors qu'elle n'a rien demandé. Elle raconte aussi qu'elle n'a eu que douze jours de congés entre le 2 juin et le 31 octobre. Elle revient aussi sur sa visite à l'inspection du travail. Son père ajoute que sa fille, aidée par un inspecteur du travail, a rédigé une lettre à son employeur afin de revendiquer des dommages et intérêts. Mauricette demande alors à jeter un œil à cette lettre ainsi qu'à celle reçue par la jeune femme de la part de la direction qui refuse de verser des dommages et intérêts. La jeune femme demande : «Ils ont le droit? De dire non?» Mauricette confirme mais précise qu'il y a d'autres possibilités, qu'ils peuvent par exemple engager une procédure au tribunal. La jeune femme reprend la parole pendant que Mauricette lit les courriers. Elle raconte que, suite à ces lettres, elle a reçu un appel de son patron lui demandant de venir discuter avec lui dès le lendemain. Lors de ce rendez-vous informel, son employeur s'est plaint de ce qu'elle n'était jamais contente, a dit qu'il ne savait pas quoi faire avec elle et que le mieux serait de signer une rupture conventionnelle, qu'elle pourrait demander elle. Mauricette ironise : «Bah oui, bien sûr ! Et en plus, c'est à vous de demander! S'il veut faire une rupture conventionnelle, il n'a qu'à la demander! Vous ne l'avez pas demandée j'espère? » La jeune femme explique qu'elle ne savait pas trop quoi faire et qu'elle n'a donc rien fait. Soulagée, Mauricette demande à son père : "Et après elle va faire quoi votre fille? Retrouver un autre emploi ?" Il répond: «Bah oui, retrouver autre chose. Parce que, si elle reste, ils vont tout faire pour la faire craquer. J'en ai bien peur!» Il demande ensuite à Mauricette : «Et si elle va voir son médecin et qu'il la met en arrêt maladie, elle a le droit ? » Mauricette lui répond : "Oui. Ce n'est pas un motif de licenciement, la maladie. À la limite, il faut aller voir la médecine du travail en leur expliquant tous vos problèmes! » Mauricette regarde alors la jeune femme et lui dit : «En plus, vous êtes travailleuse handicapée. On ne peut donc pas vous demander la même chose que les autres. Allez voir la médecine du travail. Tout ça n'est pas bon pour vous ni psychologiquement ni physiquement!»

Mauricette regarde à nouveau les documents fournis par la jeune femme et se rend compte que sa reconnaissance de la qualité de travailleur handicapé est obsolète car elle s'arrête en 2009. Elle lui demande si elle l'a renouvelée. Ni la fille ni le père ne sont capables de répondre. Ils ne savent plus. Mauricette demande alors de quoi souffre la salariée. Le père ne sait pas : «Ah ça, c'est ma femme qui sait, qui s'occupe 
de ça. » Et la jeune femme n'arrive pas à expliquer son handicap. Mauricette explique à celle-ci qu'il faut absolument qu'elle regarde si elle a renouvelé sa reconnaissance de la qualité de travailleur handicapé et que sinon il lui faut prendre rapidement contact avec la MDPH (Maison départementale des personnes handicapées). Juridiquement, elle a besoin d'un document officiel de la MDPH pour faire valoir la catégorie de travailleur handicapé.

Le père repose finalement la même question : "Bon alors qu'est-ce qu'on fait?» Mauricette répond : «La rupture conventionnelle, elle oublie! Ils ne lui ont rien dit. Elle va à la MDPH et à la médecine du travail. Elle prend rendez-vous le plus vite possible! Allez à la médecine du travail ! Je les laisse faire leur boulot ! » Mauricette donne une carte de visite à la salariée ainsi qu'au père, et elle leur demande de la rappeler une fois qu'ils auront effectué ces démarches pour reprendre un rendez-vous.

Les militants syndicaux se retrouvent donc aux prises avec des récits dont il faut comprendre le sens pour pouvoir le traduire juridiquement. Pour ce faire, il s'agit de chercher dans la mémoire ou dans la documentation juridique que s'est constituée l'union départementale des réponses au problème ainsi reconstruit. Ces réponses peuvent prendre la forme d'un calcul des indemnités ou de jours de congés pouvant être revendiqués ou encore du reclassement légitime. Pour cela, on utilise l'ensemble des outils cognitifs rendant possible ce travail de traduction.

«J'ai tout dans l'ordinateur. Je regarde, par exemple, les conventions collectives. S'il y a un problème d'interprétation de salaire minimal ou des indemnités de licenciement dans la convention $\mathrm{X}$, on prend son bulletin de paye et on a sa convention qui est clairement expliquée. On va sur la base de données et on regarde. Ici, on a tout le Code du travail. On a la jurisprudence. Les salariés regardent sur l'écran avec moi. Et parfois, je commente. Bon après, je n'en ai pas toujours besoin. Ce matin, on me demandait de calculer les jours de congé. C'est deux jours et demi par mois. On fait le calcul. On connaît les règles. On prend les bulletins de paie. On n'a pas besoin de base.»

Ce travail de traduction s'accompagne souvent d'une activité d'écriture : il est fréquent que la consultation se termine en effet par la rédaction d'une lettre à l'employeur; il n'est pas inhabituel non plus que, pour aller plus vite, le militant décide d'appeler celui-ci par téléphone. En l'occurrence, il s'agit d'aider à rétablir le contact entre salarié et employeur, ou encore (et parfois les deux en même temps) de constituer des pièces en vue de la préparation d'un dossier qui pourra être mené aux prud'hommes. Qu'il s'agisse de rédiger la lettre sur papier à en-tête du syndicat ou d'appeler directement l'employeur, c'est bien le capital symbolique de l'institution qui est utilisé comme susceptible de faire pression, voire de créer de l'inquiétude chez le chef d'entreprise. Mais c'est aussi dans le même temps une manière de rétablir une forme d'égalité entre employeur et salarié en montrant à l'employeur, dans le cadre d'un rapport de force, que celui-ci n'est plus seul et qu'il est désormais accompagné d'un spécialiste de droit du travail. 
Cette activité de consultation est ainsi d'abord un travail militant et politique de représentation des salariés, faisant du droit une ressource collective dans une logique agonistique. Si l'on s'accorde sur le fait que l'organisation syndicale ou partisane a pour objet de produire du capital politique, et si ce capital politique est bien défini par le principe d'une l'accumulation par l'organisation de ressources symboliques et collectives susceptibles d'être ensuite utilisées dans des situations individuelles pour renforcer des ressources sociales et culturelles insuffisantes ${ }^{10}$, alors les consultations juridiques constituent bien un moment proprement politique. Le syndicalisme juridique ne remet pas véritablement en cause la définition du syndicat comme institution à buts collectifs dans la mesure où le passage par le droit, même le plus individuel (celui du contrat de travail et de la fiche de paie), peut être pris dans le cadre le plus collectif ${ }^{11}$. Plus encore, ces consultations constituent en fait une sorte de solution pratique au dilemme vécu par les syndicats entre logiques collectives (de conflits ou de négociation) et défense individuelle des cas des salariés ${ }^{12}$.

Pour autant, l'activité de consultation ne nécessite pas l'usage de la « machinerie » et de la « technologie » complexe qu'analyse par exemple Bruno Latour quand il étudie le travail juridique de constitution des « dossiers» au Conseil d'État ${ }^{13}$ - et ce, même si l'apport de réponses satisfaisantes est conditionné par une maîtrise importante des règles du droit du travail. De fait, peu de militants affirment ouvrir le Code du travail ou chercher de la jurisprudence à cette occasion. Le droit qui est manipulé dans ces lieux est finalement assez faiblement formalisé. Pour autant, l'intervention des militants syndicaux ne peut pas être considérée comme un ensemble de pratiques infra-juridiques et infra-judiciaires, à la manière dont l'anthropologie historique utilise ces termes, dans le sens d'un « règlement des écarts aux normes des rapports interindividuels ou communautaires par vengeance, arrangement ou toute autre solution ne faisant pas appel aux tribunaux ${ }^{14} »$. Dans cette acception, ces termes recouvrent non seulement une non-saisie des tribunaux pour un litige, mais plus encore l'absence de perception de ce litige comme étant susceptible d'être traité dans une arène judiciaire, et donc à l'aide de catégories et d'outils juridiques. Il est

10. Bourdieu (P.), «La "variante soviétique" et le capital politique », in Raisons pratiques. Sur la théorie de l'action, Paris, Seuil, 1994.

11. Sur cette particularité du discours syndical sur le droit du travail, cf. Willemez (L.), « Le droit, un langage de la représentation. L'exemple du discours prud'homal », in Ambroise (B.), Geay (B.), dir., Langage et politique. L'efficacité du langage en question, Villeneuve d'Ascq, Presses universitaires du Septentrion, 2016.

12. C'est peut-être ce qui explique que dans l'histoire du syndicalisme, une des premières formes d'institutionnalisation a été celle des consultations au sein des bourses du travail, et ce dès le tournant du dixneuvième siècle. Sur ce point, cf. Schöttler (P.), Naissance des bourses du travail. Un appareil idéologique d'état à la fin du XIX $X^{e}$ siècle, Paris, Presses universitaires de France, 1985.

13. Latour (B.), La fabrique du droit. Une ethnographie du Conseil d'État, Paris, La Découverte, 2002, p. $83-118$.

14. Garnot (B.), dir., L'infrajudiciaire du Moyen Âge à l'époque contemporaine, Dijon, Éditions universitaires de Dijon, 1996, p. 109. 
alors sans doute préférable, dans le cas des consultations juridiques, de parler d'une activité proto-juridique, dans la mesure où ce qui se constitue et se travaille dans ces lieux, c'est la condition de possibilité d'un traitement juridique et judiciaire de difficultés et de souffrances liées au travail. De nombreux militants mettent en avant cet aspect proto-juridique de l'activité :

"Question: Et pour en revenir à votre vision du droit du travail, du Code du travail, qu'est-ce que c'est pour vous ? Qu'est-ce que ça représente ? Et à quoi ça sert ? En quoi il est utile?

Réponse: Disons que pour moi ça sert à protéger le salarié de certaines exagérations. Et puis au niveau de ses conditions de travail, avoir des conditions de travail correctes. Parce que le Code du travail, ça reprend quand même certaines choses... Par exemple, il y a un article qui me passe par la tête où l'employeur est quand même responsable de la santé physique et morale de ses employés. Donc ça, c'est quelque chose de très important !»

Une activité syndicale appuyée sur un droit assez peu formalisé aussi bien que sur une définition collective de la représentation : l'analyse de ces consultations juridiques livre ainsi une première définition du syndicalisme juridique. On comprend dans ces conditions dans quelle mesure la tâche y est d'abord de traduire l'expérience de l'injustice au travail tout en produisant une pédagogie du droit. On est alors bien en deçà de l'activité judiciaire des organisations syndicales, par exemple lorsqu'il s'agit de traiter des discriminations syndicales, qui consistent, pour leur part, à la « production d'un discours de justice cadrant la plainte » et au transport de cette plainte « au sein des arènes judiciaires » par le travail d' " acteurs collectifs qui permettent d'assurer les conditions de performativité du droit ${ }^{15} »$. Non pas que la plainte ne débouchera pas éventuellement sur ce déplacement dans l'arène judiciaire; mais le syndicalisme juridique prend ici une dimension beaucoup plus informelle et moins organisée, même s'il s'agit parfois - mais rarement en réalité - de préparer la production d'un contentieux prud'homal.

\section{"Expérience de l'injustice » et pédagogie du droit}

La spécificité de cette activité juridique s'explique principalement par le public qui est accueilli dans les lieux de consultation. Il s'agit en majeure partie de salariés issus de petites et moyennes entreprises, sans institution représentative du personnel au sein de ces entreprises, le plus souvent non syndiqués. On ne sait d'ailleurs pas très bien par quels cheminements ils viennent consulter, même si certains militants affirment que le conseil vient de l'inspection du travail. Il faut sans doute ajouter les logiques de bouche-à-oreille ou de connaissance indirecte ${ }^{16}$. Salariés (ou licenciés) de petites entreprises, les publics des

15. Chappe (V.-A.), « Dénoncer en justice les discriminations syndicales : contribution à une sociologie des appuis conventionnels de l'action judiciaire », Sociologie du travail, 55 (3), 2013, p. 304.

16. Ainsi ce salarié, rencontré lors d'une séance d'observation d'une consultation juridique : au moment où la syndicaliste sort pour aller faire des photocopies des pièces du dossier, l'enquêtrice lui demande comment 
consultations juridiques syndicales travaillent dans la vente (ce sont souvent des vendeuses dans de petites ou de grandes surfaces), dans la restauration, dans le nettoyage, etc. Ils appartiennent aux secteurs les plus précaires de l'économie française, dans lesquels les relations de subordination entre employeurs et salariés sont les plus teintées d'arbitraire.

Quoi qu'il en soit, ils sont le plus souvent sinon perdus ou du moins désorientés quand ils arrivent en consultation. Celle-ci a alors pour rôle de les aider à se repérer dans l'espace des actions possibles. Il s'agit moins de les emmener vers une procédure judiciaire que d'expliciter les enjeux et de préciser les limites que doit rencontrer l'employeur. La plupart du temps, les salariés en demande de conseil non seulement ne connaissent pas leurs droits, mais il arrive aussi qu'ils ne sachent pas lire leur fiche de paie, ne connaissent pas l'existence d'une convention collective, ou ne soient pas au courant des clauses de leur contrat de travail. Ainsi que l'affirme l'un des enquêtés :

« Nous assurons l'accueil des salariés isolés qui ont donc besoin d'un renseignement, d'un conseil, d'un appui. Et bien souvent ce sont des gens isolés. Parce que les gens qui ont une section syndicale dans leur entreprise et des élus, ils ne viennent pas à la permanence de l'union syndicale. Ce sont souvent des gens isolés, un peu paumés. Et c'est là que, moi, j'ai rencontré les situations les plus diverses et où j'ai eu plaisir à aider les gens à relever la tête ou à faire valoir ce à quoi ils avaient le droit. »

Face à ces situations d'absence totale de repères, les militants doivent d'abord et avant tout s'efforcer de trouver des réponses institutionnelles et juridiques à ce qui est le plus souvent perçu et « codé » par ceux qui demandent conseil comme un sentiment d'injustice. Les syndicalistes se retrouvent face à ce que l'on peut appeler une expérience de l'injustice dans le cadre de l' « éthique ordinaire » des relations de travail mise en avant par Emmanuel Renault ${ }^{17}$.

«C'est du passionnel. Et vous avez beau leur expliquer qu'on est dans un champ d'application du droit et que le droit du travail ne peut que compenser financièrement un préjudice, qui peut être moral mais qui ne l'est pas forcément, ils vont dire : 'Oui, mais pourquoi ? Je n’ai pas fait ci. Je n’ai pas fait ça. C’est dégueulasse !" Oui peut-être! Mais je veux dire, on n’est pas là pour dire : "Tu as fauté." On est là pour résoudre une rupture d'un contrat de travail. »

\footnotetext{
il a eu connaissance de la permanence juridique de FO. Il lui explique que ce sont des amis à lui qui lui ont conseillé d'aller voir FO bien qu'il ne soit pas adhérent, mais qu'il adhérera s'il doit aller aux prud'hommes. 17. Renault (E.), L'expérience de l'injustice. Reconnaissance et clinique de l'injustice, Paris, La Découverte, 2004. L'auteur définit ainsi cette expérience: "D’une part un sentiment d'injustice résultant de la nonsatisfaction des attentes normatives de ceux qui subissent l'injustice, d'autre part un ensemble de revendications et de tendances pratiques dirigées soit contre des facteurs identifiés comme causes de l'injustice, soit contre l'individu même qui la subit. Le concept d'expérience de l'injustice désigne donc l'injustice vécue dans sa dimension pratique et normative d'action transformatrice guidée par un sentiment; il désigne le vécu des situations justes (vécu d'injustice) accompagné d'une conscience au moins inchoative de l'injustice (sentiment d'injustice)» (p. 35).
} 
Marlène Benquet montre que l'un des ressorts de cette expérience de l'injustice est " la désignation d'une distorsion entre le fait et le droit », et qu'elle est notamment " présentée comme le résultat d'un décalage entre l'investissement dans le travail et l'absence de rétributions sous la forme de promotions ou d'obtentions de meilleures conditions de travail ${ }^{18} »$. Dans le cas des consultations, la réponse pratique à l'injustice ne tient pas dans la construction d'une mobilisation, mais plutôt d'une réponse individuelle à un salarié ou à une personne licenciée, que ce soit par un appel téléphonique ou un courrier à l'employeur, ou le renvoi vers un avocat ou l'inspecteur du travail. Ce faisant, le syndicat, par le truchement de ses militants réalisant des consultations juridiques, joue en quelque sorte un rôle de reconnaissance ou d'habilitation de ce sentiment d'injustice. En cela, les organisations syndicales peuvent être aussi définies comme des institutions de reconnaissance de l'injustice, et donc d'étiquetage de celle-ci puis de traduction en un langage, en l'occurrence ici un langage juridique.

Cette traduction part d'un discours moral et émotionnel sur ce qu'il est advenu. Il s'agit par conséquent de "gérer les sentiments » et les émotions des personnes venant chercher de l'aide ${ }^{19}$. L'enjeu est alors de les « recadrer» en mettant à distance émotion et indignation, quand bien même elle est ressentie comme parfaitement légitime par les militants. Ce recadrage nécessite alors un travail pédagogique, qui est à mi-chemin d'un travail de conscientisation au droit et de lutte contre une forme de mauvaise foi.

«Après, c'est la personne! Elle est bornée ! C'est comme ça! Mais ce n'est pas pour ça qu'on ne va pas l'aider. Mais on a des gens qui ne sont pas faciles ou des gens qui déforment tout. Moi, j'en ai eu une l'autre jour. J'étais un peu catastrophée quand elle m'a tout expliqué. Et je lui ai dit : "Quand même, j’aimerais voir vos documents et tout." Et puis, quand j'ai vu les documents, ce n'était pas du tout ce qu'elle m'avait dit. Mais ça n'avait rien à voir ! Elle avait fait une interprétation à elle, des choses qui lui étaient reprochées. Mais c'était insensé $[\ldots]$. Il n'y avait rien de ce qu'elle m'avait dit qui était marqué. Elle avait fait une interprétation et elle en était persuadée. Et elle est revenue, encore cette semaine, pour nous dire: "Je vais aller porter plainte pour diffamation. Gnagnagna !!!" J’ai été obligée de la prendre et de lui dire : "Écoutez! Je vais vous lire le courrier. Parce que, quand même, si vous l'avez lu, vous ne pouvez pas! Parce que sur quelles bases? Ça va se retourner contre vous. Donc, n'allez pas chercher les ennuis, là où il n’y en a pas." Mais il a fallu que je parle avec elle pendant deux heures, pour arriver à lui faire admettre que non, ce n'est pas la peine qu'elle aille porter plainte. Pour lui réexpliquer tout ce que je lui avais déjà expliqué avant. »

18. Benquet (M.) Les damnées de la caisse. Enquête sur une grève dans un hypermarché, Bellecombes-enBauges, Éditions du Croquant, 2011, p. 131.

19. Hochschild (A. R.), The Managed Heart: Commercialization of Human Feeling, 2nd revised edition, University of California Press, 2003. 
Un des points importants de cette activité syndicale spécifique tient donc au travail de pédagogie qui est réalisé. Par ce que l'on pourrait appeler une forme d'éducation populaire, il s'agit d'apprendre aux individus venus demander de l'aide la manière de faire valoir leurs droits. Cela passe donc par une aide à la lecture des documents qu'ils reçoivent, à des informations sur le droit du travail, voire à des décodages de la langue juridique. Mais au-delà, l'objectif est surtout de les aider à surmonter morale et émotion pour faire place à un discours de rationalité. Mais tel n'est-il pas au fond ce que prétendent faire tous les militants syndicaux ? Donner aux salariés les moyens de se défendre contre les empiétements des directions d'entreprise, permettre l'expression d'une parole contradictoire : ces activités constituent en réalité le quotidien du travail syndical. Mais ceci ne peut se faire sans que se déroule dans le même temps une logique d'imposition par les militants de catégories socialement acceptables pour penser la réalité sociale, ici les relations de travail. Le fait que ces outils soient juridiques et judiciaires ne change finalement pas grand-chose à l'affaire, dans la mesure où ils ne sont pas encore " décollectivisés " par le passage entre les mains des professionnels du champ juridique. Contrairement à d'autres aspects du syndicalisme juridique susceptibles de transformer les pratiques syndicales, les consultations juridiques apparaissent donc comme une forme peu discutable de l'intervention syndicale à partir d'outils juridiques.

\section{Une activité juridico-syndicale entre marginalité et centralité}

De fait, les acteurs de ces consultations sont bien intégrés dans le monde juridico-syndical, surtout au niveau local et départemental. Leur activité de consultation juridique n'est qu'un des aspects de ce qui apparaît comme une véritable carrière : ces militants sont souvent des conseillers prud'hommes actuels ou anciens, ainsi que des conseillers du salarié ${ }^{20}$. En tout état de cause, ils sont des personnages centraux de l'interprofessionnel, dans la mesure où, comme nous l'avons montré par ailleurs, l'activité juridique est une des formes de légitimation de ce niveau syndical. Le responsable d'une union locale explique par exemple :

«Certains ont plusieurs casquettes. Pierre est conseiller prud'homme. Jérémy est conseiller du salarié. Mauricette est conseiller du salarié. Jacques ne fait que cela actuellement. Élisabeth est une déléguée syndicale centrale dans le commerce non alimentaire. Voilà. [Il réfléchit et regarde le planning qu'il m'a montré tout à l'heure.] Je suis en train de regarder. Luc est un ancien conseiller prud'homme. On est sur des personnes qui ont eu plusieurs casquettes. Il en manque d'ailleurs. Patrice est un ancien directeur de ressources humaines. [...] Ils ont eu des responsabilités syndicales qui les ont amenés à un moment donné

20. Le conseiller du salarié assiste ce dernier lors de l'entretien préalable au licenciement avec son employeur ou son représentant. Pour une analyse de cette figure peu connue, cf. Ferrette (J.), «Au-delà de l'action directe et de l'institutionnalisation, les conseillers du salarié », Travail et emploi, 122, 2010. 
à côtoyer l'union départementale régulièrement. Et on le sait tous, c'est d'abord et avant tout la relation et la confiance, la relation humaine et la confiance entre les individus qui amènent parfois à partager des discussions de cette nature. »

On comprend alors que l'étude des rapports au droit et de la socialisation de ces militants ressort du même type d'analyse que pour les conseillers prud'hommes. On y retrouve les mêmes «identités juridiques hétérogènes ${ }^{21}$ ", qu'il s'agisse de militants autodidactes, non diplômés et investissant une grande partie de leur temps dans l'activité juridique, ou de militants ayant un "goût » pour le droit et s'y spécialisant, ou encore de professionnels ou d'anciens professionnels du champ juridique vivant leur activité de conseil juridique et de défense judiciaire dans la continuité de leur activité professionnelle actuelle ou précédente.

Le fait qu'il s'agisse d'un même groupe d'individus multipliant les rôles juridiques et judiciaires permet d'éclairer sous un autre jour les formations juridiques dont bénéficient les conseillers prud'hommes, et qui sont financées par l'État mais assurées par des organismes de formation internes aux organisations syndicales et patronales. En réalité, elles ne forment pas seulement les conseillers prud'hommes à leur rôle de juge, mais aussi aux autres fonctions de défense juridique des salariés, et donc en particulier au travail de consultation juridique.

«Ça fait plusieurs dizaines d'années, en fait, que je tiens cette permanence. J'ai été près de trente ans conseiller prud'homme. Donc c'est parti de là, en fait! Quand j'ai acquis un minimum d'expérience, j'ai un peu orienté aussi la permanence vers ça. Et c'est aussi une des choses qui est prise en charge par le syndicat pour tous ses adhérents : le soutien juridique. Ça s'est structuré avec le temps, avec la généralisation des conseils de prud'hommes, où là il y a eu des conseillers prud'hommes plus nombreux qui se sont formés avec le temps et qui sont devenus un peu les référents de tout ce qui est accès au droit, un peu technique. Autrement, ce sont les élus du personnel, les militants d'entreprise qui peuvent aider leurs collègues, les informer, les soutenir dans une démarche en interne. Au niveau interprofessionnel, ça s'est mis en place en appui sur les équipes de conseillers prud'hommes, qui eux avaient accès statutairement à la formation et qui par leur fonction travaillaient la matière de façon beaucoup plus soutenue que la plupart des militants. »

L'activité de consultation juridique peut ainsi apparaître comme une pratique tout à la fois marginale et centrale dans l'ensemble des formes que prend le syndicalisme juridique. Elle est marginale dans la mesure où elle est exercée par des militants ayant d'autres activités juridiques plus valorisantes (l'activité

21. Willemez (L.), «Activités judiciaires et trajectoires juridiques. Les conseillers prud'hommes et le droit du travail », in Michel (H.), Willemez (L.), dir., Les prud'hommes. Actualité d'une justice bicentenaire, Bellecombe-en-Bauges, Éditions du Croquant, 2008. 
prud'homale par exemple) ou pour qui elles constituent une manière de contredon, et parce qu'elle est routinière et renvoie à un état du droit peu formalisé et particulièrement indexé sur la pratique. Mais elle est en même temps centrale au sens où elle est une des formes d'action les plus légitimes de la défense syndicale, renvoyant les organisations à leur histoire via les bourses du travail, et parce qu'elle constitue une des raisons d'être des unions locales ou départementales. Enfin, elle est susceptible de participer à l'effort de syndicalisation, entendue comme le développement de l'adhésion aux organisations en direction de publics qui étaient jusque-là éloignés d'elles : salariés des très petites entreprises ou des petites et moyennes entreprises, travailleurs précaires de toutes sortes, étrangers, voire sans-papiers ${ }^{22}$.

\section{Entre activité bureaucratique et engagement politique : les " renseignements " juridiques de l'inspection du travail comme activité de guichet}

Une comparaison avec un autre type de consultations, celles qui ont lieu à l'inspection du travail, permet de montrer en creux la spécificité des conseils en droit du travail issus du monde syndical.

\section{Un service public du conseil juridique en droit du travail}

Les agents de contrôle du travail (c'est ainsi que l'on nomme le corps des contrôleurs - agents de catégorie B - et inspecteurs du travail - catégorie A) exercent donc une mission de service public qualifié de « renseignements ». Ils participent pleinement à ce que Pierre-Yves Baudot et Anne Revillard appellent une " politique des droits ", dans la mesure où ils contribuent à " une défense des droits à l'intérieur du processus administratif ${ }^{23} »$. Cette partie du travail de ce corps de fonctionnaires n'est évidemment pas la plus connue, les tâches d'inspection ayant été beaucoup plus mises en avant, et par exemple la contribution des inspecteurs à l'émergence des questions de santé au travail ${ }^{24}$. Il reste que les agents de contrôle ont bien cette autre mission, qui consiste à écouter les doléances des salariés pour leur donner des conseils juridiques. Cette activité s'appuie sur deux types de permanences, l'une téléphonique et l'autre physique, sur les différents sites de l'inspection du travail. Celle-ci est appelée de « renseignements » dans l'organisation bureaucratique des directions départementales

\footnotetext{
22. Pour une évocation des consultations juridiques qui ont accompagné les grèves de travailleurs sans papiers à la fin des années 2000, cf. Baron (P.), Bory (A.), Chauvin (S.), Jounin (N.), Tourette (L.), « On bosse ici, on reste ici! " La grève des sans-papiers : une aventure inédite, Paris, La Découverte, 2011. D’une manière plus générale, cf. Béroud (S.), « Organiser les inorganisés », Politix, 85, 2009.

23. Baudot (P.-Y.), Revillard (A.), «Une sociologie de l'État par les droits », in Baudot (P.-Y.), Revillard (A.), dir., L'État des droits. Politique des droits et pratiques des institutions, Paris, Presses de Sciences Po, 2015, p. 34-35.

24. Tiano (V.), «Déficit des régulations et perte de légitimité d'une profession : les inspecteurs du travail », in Action publique et légitimités professionnelles, Paris, LGDJ-Lextenso, 2008.
} 
du travail (où se déroule l'activité), même si la convention n ${ }^{\circ} 81$ de l'Organisation internationale du travail (OIT), qui encadre l'activité des inspecteurs du travail, stipule que le « système d'inspection du travail sera chargé » notamment " de fournir des informations et des conseils techniques aux employeurs et aux travailleurs sur les moyens les plus efficaces d'observer les dispositions légales ». Le rôle est cependant ambigu dans la mesure où il peut entrer en contradiction avec le rôle de surveillance et de sanction qu'ont habituellement les conseillers, et surtout qu'il s'adresse à l'ensemble des acteurs du monde du travail, salariés et employeurs. Il s'appuie donc sur une déontologie définie par un document publié en 2010 par la direction générale du travail, selon lequel :

"Les conseils techniques recouvrent l'ensemble du champ de la législation du travail et vont de l'explication de la règle et des modalités de sa mise en œuvre jusqu'à l'orientation vers d'autres organismes, institutions ou experts. Il ne s'agit pas pour l'agent de contrôle de déterminer à la place du demandeur la solution technique ou/et juridique, mais seulement de fournir des indications propres à respecter la règle ${ }^{25}$.»

Les agents de contrôle sont donc pris dans des contraintes juridiques - en l'occurrence déontologiques - assez fortes, avec lesquelles ils doivent composer pour endosser le rôle. Le paradoxe est sans doute que c'est précisément l'existence de ces contraintes institutionnelles qui permet à certains d'entre eux d'investir des enjeux militants dans cette activité et de jouer avec les limites du rôle, qui sont imposées mais aussi négociées au jour le jour, dans les moments d'interaction que sont les consultations ${ }^{26}$.

Le travail proprement dit de conseil au public, et en réalité très largement aux salariés, se tient concrètement à travers les permanences organisées dans les unités territoriales de l'inspection du travail. Elles sont réalisées par des contrôleurs du travail dédiés à ce service, dont c'est la tâche unique et prenant l'ensemble de leur temps de travail, et qui sont appelés agents du service de renseignement. Dans le département dans lequel la recherche a été effectuée, ils sont deux à exercer cette activité ${ }^{27}$. Si l'on n'a pas eu accès au nombre total de renseignements donnés au cours d'une année, le chiffre est d'environ 42000 pour l'ensemble de la région en $2011^{28}$.

25. Direction générale du travail, Principes de déontologie pour l'inspection du travail, en ligne :http://www. intefp-sstfp.travail.gouv.fr/datas/files/SSTFP/D\%C3\%A9ontologie\%20Inspection\%20du\%20travail\%20 25\%2002\%2010.pdf, consulté le 11 mars 2017.

26. Lagroye (J.), Offerlé (M.), dir., Sociologie de l’institution, Paris, Belin, 2011.

27. Contre, par exemple, sept dans les Yvelines.

28. Pour donner un ordre de grandeur, le bilan annuel de la Direction générale des entreprises, de la concurrence, de la consommation, du travail et de l'emploi (DIRECCTE) de l'île-de-France pour 2011 indique que les services de renseignements des unités territoriales des sept départements ont reçu 45000 usagers et répondu à 112000 appels téléphoniques. De son côté, le bilan de l'inspection du travail sur l'ensemble de la France fait état pour la même année de 7,9 millions de " conseils donnés au public », selon la terminologie du rapport. 
Ces données permettent d'établir des indicateurs de l'activité conformes à la diffusion des outils du nouveau management public. Comme tous les services publics, les consultations juridiques sont touchées par les transformations de l'État et par l'amoindrissement des tâches de service public qui les ont accompagnées. Dans le département dans lequel nous avons enquêté, l'amplitude horaire des consultations a été divisée par deux. Désormais, c'est seulement sur cinq demi-journées par semaine (au lieu de cinq jours entiers) que les contrôleurs du travail reçoivent les usagers. De même, ils ne sont pas remplacés en cas de maladie ou de simple congé, et c'est donc l'ensemble du service qui tourne à demi. Les inspecteurs et contrôleurs du travail font le même constat que de nombreux autres agents de la fonction publique quant à la difficulté de réaliser leurs missions, qu'elles soient de contrôle ou ici d'information et de conseil.

« Le salarié quand il appelle, il n’y a plus personne pour lui répondre, on n’est plus assez, les collègues font ce qu'ils peuvent, les demandes d'intervention, c'est le cœur même du métier, mais des demandes d'intervention, il y en a plein, des salariés qui écrivent, qui ne sont pas contents, parce qu'il y a personne qui vient... Mais il faut hiérarchiser, il faut... On ne peut pas... Après c'est un choix politique qui ne nous appartient pas. »

C'est ainsi le double processus d'une part de dégradation budgétaire et son accélération ces dernières années, et d'autre part de réorganisation de l'État autour d'un modèle managérial, qui est vécu douloureusement par l'ensemble des agents du ministère du Travail. Ceux-ci ressentent une vive « expérience de la dépossession ${ }^{29} »$ de leur métier et de la manière dont ils le pratiquent - ce qui renvoie en réalité aux valeurs qu'ils défendent et qu'ils investissent dans la manière d'exercer leur rôle professionnel.

Il faut néanmoins ajouter que des conseils sont donnés à d'autres moments par les agents de contrôle. C'est notamment le cas au moment de la mission de contrôle elle-même, ou encore par téléphone, ou dans le bureau même de l'inspecteur : celui-ci consacre souvent une demi-journée par semaine à recevoir ou à appeler les individus concernés par leur contrôle, qu'il s'agisse de représentants du personnel, de directeurs des ressources humaines ou encore de petits chefs d'entreprise qui se renseignent sur les procédures.

«Les personnes qu'on reçoit... Moi j'en reçois pas mal parce que j'essaie de sortir pas mal donc je convoque beaucoup d'employeurs déjà, puis après les salariés, tu en as beaucoup qui viennent te voir, tu as les représentants du personnel qui viennent te voir, parce que c'est un maillon essentiel, parce que toi tu ne peux pas tout voir, donc les délégués du personnel, c'est avec eux que tu... Tu leur donnes des armes, tu les outilles, tu leur donnes des armes pour mener à bien leur mission quoi, et puis eux, ils sont sources d'infos pour toi, ils te disent, voilà... [...] Moi je reçois le jeudi, mais tu as des salariés qui ne peuvent pas venir bien évidemment le jeudi matin, après on s'arrange. »

29. Ogien (A.), Laugier (S.), Pourquoi désobéir en démocratie?, Paris, La Découverte, 2010. 
En réalité, cette activité de conseil semble beaucoup plus courante que la sanction proprement dite, qui passe par un procès-verbal envoyé au procureur de la République. Contrairement aux représentations que l'on peut s'en faire, les inspecteurs du travail se considèrent - et sont considérés de plus en plus par leur hiérarchie - comme des agents chargés de rappeler le droit et de conseiller les employeurs dans les conditions de l'application du droit du travail. On retrouve ici un important aspect de pédagogie du droit, qui est finalement assez éloigné de la construction historique du rôle des inspecteurs du travail.

\section{Du conseil au filtrage}

Plus que pour les consultations juridiques dans les organisations syndicales, on est ici largement dans une logique de guichet, l'activité de conseil mettant aux prises un fonctionnaire et un usager, ici un demandeur de conseils. Pour autant, leur activité les rapproche dans le même temps de leurs « confrères » des consultations syndicales.

En premier lieu, les contrôleurs du travail exercent, comme les syndicalistes, une véritable activité de pédagogie auprès des salariés venus demander de l'aide. Ils expliquent et réexpliquent les procédures liées au licenciement, en amont (en particulier à travers l'entretien préalable au licenciement et le droit d'y être assisté) comme en aval, notamment autour du contrat de sécurisation professionnelle (CSP), qui était au moment de l'enquête la mesure phare des politiques d'accompagnement des licenciements collectifs. Comme les syndicalistes, ils aident au calcul des indemnités que les salariés pourraient obtenir ; ils expliquent les enjeux, les intérêts et les contraintes de la rupture conventionnelle; ils reviennent sur les questions de temps de travail, en particulier en termes de temps de travail atypique, etc. Pour ce faire, les contrôleurs vont chercher sur Internet le Code du travail et les conventions collectives; ils usent aussi volontiers de la calculatrice (pour calculer les indemnités et les primes de licenciement), réalisent des schémas pour expliquer des calculs ou des procédures, ou encore rédigent des brouillons de lettres - ce qui d'ailleurs, on le verra, pose un certain nombre de problèmes éthiques.

\section{Encadré 3. Une consultation juridique auprès des contrôleurs du travail}

Soit l'exemple d'une observation réalisée lors d'une permanence : une coiffeuse vient de recevoir une lettre de convocation à un entretien de licenciement. C'est le nouveau repreneur de l'entreprise qui la licencie. Xavier, le contrôleur, lui explique qu'elle a le droit de se faire accompagner par un conseiller du salarié ; il lui fournit la liste des conseillers du salarié disponibles et lui explique comment ça fonctionne, en lui conseillant d'appeler les unions départementales des syndicats pour trouver la personne adéquate. Mais la salariée veut savoir le montant de la prime qu'elle va à peu près toucher. Il lui explique le mode de calcul : pour mesurer l'indemnité 
de licenciement, il faut calculer l'ancienneté. Pris d'un doute, il va vérifier dans le Code du travail la manière d'intégrer le congé parental dans l'ancienneté : selon l'article 1225-54, elle va perdre un peu d'ancienneté. Il lui fait même un petit schéma pour expliquer en quoi va consister l'indemnité. En fait, la salariée avait trouvé sur Internet le mode de calcul, qu'elle veut lui faire vérifier : « Votre mode de calcul est le bon. » Il lui explique la procédure que constitue le CSP, ses avantages et ses inconvénients, de manière à ce qu'elle choisisse en connaissance de cause. La discussion porte ensuite sur l'acceptation du licenciement. Xavier explique à la salariée qu'en l'espèce, il n'est guère possible de discuter la décision. Certes, "on peut toujours aller devant le conseil des prud'hommes », mais il faut se demander pour quoi faire: dans cette procédure de licenciement pour motif économique, « les prud'hommes, ça ne sert pas à grand-chose ». On comprend qu'en réalité, il la pousse plutôt à ne pas contester.

Ce travail pédagogique apparaît comme particulièrement routinier. Lors des séances d'observation, les échanges semblaient plus répétitifs et plus bureaucratiques que de la part des militants syndicaux ${ }^{30}$. Mais à la différence de ces derniers, les contrôleurs sont apparus plus fermes dans l'explicitation de l'espace des possibles pour les salariés : ils passent du temps à en dissuader certains, en montrant qu'il n'y a rien à faire, et ce, alors même que ceux-ci viennent en réalité mesurer s'il vaut la peine qu'ils «tentent leur chance », et ce même s'ils ne connaissent pas véritablement les outils qui pourraient les aider.

De fait, on a le sentiment que les contrôleurs du travail ont affaire à des salariés détenant plus de capital culturel, ou du moins étant plus en mesure d'aller chercher des informations, en particulier sur Internet. Ils cherchent d'abord des garanties institutionnelles aux stratégies qu'ils veulent mettre en œuvre et aux réponses qu'ils veulent apporter face à l'employeur. Les contrôleurs se retrouvent alors dans un rôle de légitimation institutionnelle d'une stratégie juridique. Même si les salariés venant dans les consultations ne sont pas tous pourvus de ce type de connaissances juridiques et de capacités à obtenir des informations, il reste que l'aspect institutionnel de la démarche qui consiste à aller à l'inspection du travail fait que ces salariés sont sans doute moins démunis juridiquement et socialement que ceux qui viennent aux permanences syndicales. En tout état de cause, ils expriment beaucoup moins d'indignation morale et de sentiment d'injustice, comme si un premier travail de traduction avait été réalisé par le choix de passer par le filtre institutionnel, ou a contrario comme si la démarche syndicale s'appuyait sur ce moment d'expression morale et émotionnelle.

30. Mais cela est peut-être dû au fait que le nombre relativement réduit d'observations ne permettait guère de comprendre les variations entre les différents acteurs des consultations. 


\section{Encadré 4. Une consultation juridique auprès d'un contrôleur du travail}

Xavier reçoit un commercial qui vend de l'optique : celui-ci est très mécontent car son employeur modifie souvent son secteur géographique d'activité ainsi que le catalogue de ce qu'il vend. En l'occurrence, l'employeur modifie périodiquement la collection de lunettes qu'il vend, et il lui a notamment confié dernièrement des montures moins prestigieuses, ce qui lui a fait perdre de l'argent (on comprend ces éléments au fur et à mesure). Le contrôleur traduit immédiatement la demande du commercial en une question juridique : y a-t-il modification du contrat de travail ou modification des conditions de travail ? Dans le premier cas, le salarié peut attaquer son employeur aux prud'hommes, mais pas dans le second. Pour comprendre cela, il faut lire le contrat de travail, qui stipule non seulement que l'employeur peut changer le commercial de secteur d'activité, mais qu'en outre celui-ci a un fixe et une prime de résultats : " Je vais regarder si vous êtes dans un cas de modification du contrat de travail. » Xavier réfléchit longuement, dans un silence qui dure plusieurs dizaines de secondes, lisant le contrat de travail et consultant le Code du travail et la convention collective des VRP, mais manifestement il ne trouve rien qui puisse aider le commercial. Au bout d'un certain temps, le salarié intervient : "Il y a une jurisprudence, je crois. » Il lit le texte avec Xavier sur l'écran, explique sa vision des choses et revendique son bon droit. Toujours très patient et extrêmement calme, le contrôleur montre que la jurisprudence ne s'applique pas : "Dès lors qu'ils vous garantissent un fixe lié à la vente, il n'y a pas grand-chose à faire. Malheureusement, je ne vois pas ce qu'on peut faire : l'aspect variable de la rémunération est là. Pour le coup, je ne peux pas faire grand-chose ; je n'ai aucun fondement juridique pour le dire. » Le commercial repart déçu, et Xavier fait ce commentaire à l'enquêteur : « Il gagne déjà beaucoup d'argent. Lui, il a son fixe qui est respecté... Le reste, le risque, c'est lié à son métier.»

Ainsi, comme pour les avocats, l'activité de consultation est susceptible de constituer un travail de filtrage des demandes. En cela, on pourrait considérer que les agents de contrôle exercent une véritable fonction de sélection des causes pouvant relever des juridictions. On retrouve alors les mêmes types d'énervement contre les usagers pénibles, venus demander justice avec un aplomb qui paraît hors de propos dans la mesure où ces acteurs utilisant le droit au quotidien ne peuvent accepter que l'on considère la justice dans une perspective purement stratégique :

«Avec Christine, notre secrétaire, on échange et ça fait qu'elle connait, elle sait. Il y a des fois elle sait qu'untel, c'est bon quoi! Parce que t'as des emmerdeurs aussi, bon. T'as ceux qui vont t'embêter, qui vont t'appeler trois fois par semaine parce que c'est l'affaire du siècle, parce que tu leur as répondu, mais eux, ils ne sont pas forcément d'accord sur la réponse, mais que ceci, mais que cela... Donc elle sait aussi filtrer, elle sait aussi voir l'intérêt. »

Tout cela permet de montrer que les inspecteurs du travail constituent bien une profession juridique, même si la littérature scientifique les présente assez 
peu de cette manière, préférant voir en eux des acteurs du monde des relations professionnelles. Par leur activité, par leur formation aussi, ils sont plus que des intermédiaires du droit, dans la définition que Jérôme Pélisse en donne de « non-juristes de profession qui le rencontrent au quotidien dans leurs activités professionnelles $^{31}{ }^{\prime}$, dans la mesure où on peut considérer leurs pratiques de travail comme le maniement quotidien d'outils et de catégories juridiques ${ }^{32}$. Mais dans le même temps, leur fonction ressemble fortement à celle des militants syndicaux, dans la mesure où leur travail de renseignement volontiers " proto-juridique» (comme le montrent la rédaction de brouillons de lettres ou la réalisation de calculs d'indemnités). Enfin, ils sont beaucoup plus pris dans des logiques institutionnelles qui les contraignent tout en leur offrant une forte légitimité. Ceux-ci sont en effet marqués du sceau de l'État, qui donne aux contrôleurs du travail le capital symbolique leur permettant de créditer les conseils qu'ils fournissent de l'autorité symbolique que celui-ci leur confère ${ }^{33}$.

\section{Une activité de conseil inscrite dans un ethos professionnel}

Dans les entretiens, les inspecteurs et contrôleurs du travail insistent très souvent sur trois valeurs combinées qui orientent leurs actions : le service public, le droit et leurs positions politiques. En premier lieu, l'importance centrale prise par la «mission » de service public est sans cesse rappelée par ces agents, pour qui le service de l'État constitue la raison d'être de leur activité et le cœur de leur identité sociale et professionnelle. Mais ce sens du service de l'État est immédiatement lié à un autre aspect des valeurs, celui des « convictions » ou encore de la «fibre sociale» des agents. Cette «fibre sociale» prend en fait un sens particulier, celui d'un engagement politique et social aux côtés des salariés dans le cadre de la relation salariale considérée comme asymétrique.

«On est viscéralement attaché à cette mission de service public, c'est chevillé au corps, c'est quelque chose, encore une fois, je pense, sans aucune prétention, mais vraiment aucune - je veux que ce que je dis reste anonyme -, mais je pense que pour certains agents, c'est vraiment un travail militant mais au sens... On est neutres hein, on a une obligation de neutralité en étant agent de l'État et encore plus en section d'inspection du travail... Mais travail militant au sens

\footnotetext{
31. Pélisse (J.), Le travail du droit..., op. cit.

32. C'est d'ailleurs toute la thèse de l'ouvrage de L. Justet : L'inspection du travail: une expérience du droit, Rennes, Presses universitaires de Rennes, 2013. La plupart des (rares) travaux existants insistent plutôt sur le rôle des inspecteurs du travail dans la prévention des risques professionnels. Pour une exception, cf. Pélisse (J.), «Inspecteurs et jeux d'acteurs. Les avatars du contrôle du temps de travail depuis les années 70 », in Le Crom (J.-P.), dir., Les acteurs de l'histoire du droit du travail, Rennes, Presses universitaires de Rennes, 2004. 33. On peut rappeler ici la définition que P. Bourdieu donne de l'action de l'État, dont l'un des rôles est d'« instituer des différences entre les consacrés et les non-consacrés » : "Ce sont des différences qui sont de l'ordre de la magie sociale, et qui sont produites par un acte de constitution instaurant des divisions durables, définitives, indélébiles, souvent insurmontables parce qu'elles sont sans cesse rappelées aux corps par le monde social » (Bourdieu (P.), Sur l'État. Cours au Collège de France (1989-1992), Paris, Seuil/Raisons d'agir, 2012, p. 267).
} 
noble du terme, c'est-à-dire que pour certains je pense, la majorité, on n'est pas là par hasard et on fait ce travail avec vraiment des convictions profondes. C'est ce qui nous permet de tenir, parce que c'est un travail qui, malgré l'autonomie, la liberté, etc., qui est pas si facile que ça.»

On voit bien ainsi les contradictions inhérentes à la position de ces agents. En premier lieu, le sens du service public est « chevillé au corps », et les inspecteurs du travail proposent une image d'eux-mêmes comme proche de la figure sociale de l'instituteur républicain. D'ailleurs, on ne peut que faire le parallèle entre les "hussards de la République » et les "voltigeurs de la République », expressions toutes deux forgées au début du $\mathrm{XX}^{\mathrm{e}}$ siècle pour marquer l'ethos républicain du métier au sens d'un engagement sacrificiel au service de l'État et du bien commun, que ce soit pour éduquer le peuple ou pour contrôler le monde du travail ${ }^{34}$.

Mais cet extrait d'entretien pointe aussi l'exigence de neutralité liée à l'appartenance à la fonction publique. Cette exigence est martelée dans les ouvrages formant les inspecteurs du travail, mais aussi dans les propos des responsables hiérarchiques. Les proclamations d'objectivité et de neutralité constituent une expression extrêmement classique d'une représentation majeure de la fonction publique, et renvoient à l'idéal-type de la bureaucratie telle qu'a pu la définir Max Weber ${ }^{35}$ : spécialisation dans les tâches, technicité et compétence, autonomie et dépendance unique à l'État, etc. C'est aussi l'enjeu même des limites administratives portées au travail de conseil juridique, dont on a vu qu'elles encadraient assez strictement le rôle. La réalité est beaucoup plus complexe, et les protestations de neutralité des agents de contrôle peuvent apparaître au mieux comme un paravent, au pire comme un faux-nez. Ce serait pourtant une erreur dans la mesure où ces représentations officielles du métier constituent bien une base à partir de laquelle les agents pensent leur rôle et définissent leurs pratiques. Dans ce cadre, Vincent Dubois analyse l'existence des « deux corps du guichetier », comme Ernst Kantorowicz a évoqué les « deux corps du roi ${ }^{36}$ ». Agent de l'État, le fonctionnaire, guichetier à la caisse d'allocation familiale ou agent de contrôle du ministère du Travail, est en même temps une personne physique, dotée de dispositions liées à son appartenance sociale, à ses positions politiques, au rapport qu'il a à son poste et à sa profession, etc. Dans son analyse des guichetiers, Vincent Dubois montre que «c'est dans la gestion des écarts [sociaux entre les guichetiers et les usagers] que se jouent les conditions du repli

34. Pour les inspecteurs du travail, cette conception du métier s'ancre en effet dans une histoire longue: cf. Viet (V.), Les voltigeurs de la République. L'inspection du travail en France jusqu'en 1914, Paris, CNRS Éditions, 1994. Il en est de même pour les instituteurs. Sur l'ethos professionnel de ces derniers et sa genèse, cf. Geay (B.), Le syndicalisme enseignant, Paris, La Découverte, 1997.

35. Weber (M.), La domination, Paris, La Découverte, 2013, p. 63-118.

36. Dubois (V.), La vie au guichet. Relation administrative et traitement de la misère, Paris, Economica, 2005, p. 81-146. 
bureaucratique ou de l'engagement individuel, de l'application formaliste de la règle ou de la mobilisation des ressources personnelles ${ }^{37}$ ». C'est à partir de cette double position que les agents de contrôle du ministère du Travail réalisent leurs activités de conseil.

À ces deux points de vue s'en ajoute d'ailleurs un troisième, celui du droit. Comme on le sait, la force du champ juridique est de mettre à distance les profanes tout en imposant des modes de régulation considérés sinon comme naturels ou universels, du moins comme des évidences non questionnables ${ }^{38}$. Pour les inspecteurs du travail (comme pour des avocats militants, des magistrats ou des enseignants-chercheurs en droit engagés dans l'activité syndicale), le droit peut aussi, par ce fait même, constituer une arme susceptible de minimiser le déséquilibre lié à la relation de subordination dans le contrat de travail. La croyance dans ces deux caractéristiques propres au droit constitue donc une autre valeur sur laquelle ils appuient leur action.

"C'est un travail militant, je pense que tu comprends le sens, pas un côté contre l'autre, non, nous le seul parti qu'on doit prendre c'est le droit mais... Sans faire des grands mots, le droit du travail existe parce que la relation de travail est déséquilibrée, hein. Et c'est pour ça que le droit du travail s'est construit. Nous, notre quotidien, c'est toute la journée d'essayer de rééquilibrer. Justement, de voir des gens qui sont en souffrance ou dans la détresse, qui sont pas reconnus dans leur droit, et c'est notre but. Donc on n'est pas là pour prendre parti pour, on est là pour faire appliquer une loi. Donc si t’as pas la conviction, si t'as pas un tout petit peu une fibre sociale euh... J'vois pas... Ma conviction profonde, pour faire ce travail, c'est qu'il faut quand même avoir un engagement, une fibre sociale importante.»

\section{Des consultations ambiguës}

Cette pluralité de valeurs s'actualise bien entendu dans les consultations juridiques, comme d'ailleurs sans doute dans l'ensemble des activités des inspecteurs du travail. La définition officielle de ce que doit être ce travail de consultation est ainsi proposée par un directeur-adjoint du travail.

«On ne donne que la règle de droit [...] On répond sur le droit. Notamment, il n'y a pas de contrôle de documents, pas de contrôle de la fiche de paie par exemple: on ne fait pas de calculs; et donc on ne donne pas vraiment de conseils, en tout cas pas de conseils stratégiques. Certes, il existe des situations choquantes, mais l'on s'oblige à ne pas conseiller. Même quand des situations sont moralement choquantes; par exemple quand une vendeuse a une amplitude de travail extrêmement étendue, alors même qu'elle ne travaille que quatre heures par jour. Mais là, on ne peut rien faire, on ne peut rien dire. On ne dit rien.»

37. Ibid., p. 118.

38. Bourdieu (P.), « La force du droit », Actes de la recherche en sciences sociales, 64, 1986. 
On voit particulièrement bien ici l'écart très important entre le discours officiel et la réalité de la pratique telle que nous avons pu l'observer. Le premier conduit à interdire jusqu'au terme même de conseil, conformément au cadre juridique. Les agents de contrôle sont ainsi pris dans une double contrainte juridique, celle du droit du travail (c'est-à-dire le Code du travail et la jurisprudence) et du droit qui entoure leur mission de service public. L'extrait d'entretien qui suit le montre particulièrement bien :

« La marge de manœuvre, elle est réduite, on peut avoir les convictions les plus profondes qui soient, on n'en demeure pas moins fonctionnaire d'État avec un cadre juridique strict, donc il est évident qu'on ne peut pas faire tout et n’importe quoi. Néanmoins, dans la manière d'aborder les problèmes, dans la manière de renseigner les gens, dans la manière de renseigner les gens, dans la manière de déborder plus ou moins du cadre juridique qui nous est donné, il est bien évident que dans la manière d'exercer le métier, les convictions personnelles de chaque agent entrent en ligne de compte.»

Ce qui nous renvoie au jeu avec les règles qui est permis à ces professionnels du droit, virtuoses dans ce que Jean-Pierre Le Bourhis et Pierre Lascoumes ont appelé les " passes du droit ${ }^{39}$ » et qui constitue le travail ordinaire d'une partie des fonctionnaires, ceux du moins dont les dispositions sociales, politiques et professionnelles les y autorisent. Comme montre l'extrait d'entretien qui vient d'être cité, c'est bien l'activité même, «la manière de déborder plus ou moins du cadre juridique qui nous est donné », qui constitue un espace de résolution pratique des dilemmes professionnels et politiques des agents de contrôle. C'est ce qui explique que les réponses aux questions des salariés sont souvent précédées de longs moments de réflexion, qui correspondent certes au travail de traduction d'expériences vécues de l'injustice en catégories juridiques, mais qui sont aussi le moment d'une délibération intérieure sur l'opportunité et la façon de franchir des limites, de dépasser ou non les contraintes imposées, de mesurer le besoin qu'ont les salariés quant au franchissement de ces limites.

Ces moments ne sont d'ailleurs pas nécessairement perçus comme des délibérations ou des « colloques intérieurs » car ils appartiennent en réalité au sens pratique professionnel des inspecteurs du travail ${ }^{40}$. Un autre extrait d'entretien avec le même agent permet de montrer de quelle manière ce sens pratique professionnel est différencié selon les publics et spécifie un mode particulier de conseil :

« Tiens, là, je l'ai pas fait exprès, mais il traîne sur mon bureau, voilà un dossier, je vais pas rentrer dans les détails car il est extrêmement compliqué. Mais là, je suis allé bien au-delà... Alors moi je le fais, mais quantité de collègues le

39. Lascoumes (P.), Le Bourhis (J.-P.), « Des passe-droit aux passes du droit. La mise en œuvre socio-juridique de l'action publique », Droit et société, 32, 1996.

40. Bodin (R.), « Une éducation sentimentale », Déviance et société, 35 (1), 2011. 
font, je suis allé bien au-delà de mes prérogatives, en tout cas de ce que devrait être mon rôle, et j'ai pour ainsi dire écrit le courrier que la salariée a envoyé à l'employeur, parce que c'était une personne qui avait des difficultés à écrire et à lire, donc ce travail-là qui devrait normalement ne pas être le nôtre mais plutôt celui des syndicats, des représentants du personnel, mais lorsqu'on est, comme c'est le cas là dans des micro-entreprises ou en l'occurrence, le patron a des pratiques, c'est à la limite de... L'expression "patron voyou” est en l'occurrence tout à fait adaptée. Voilà les salariés sont complètement démunis, ils se retournent vers nous. Moi, si je lis le texte, si je lis le code, tout ce que réclame la salariée, c'est de la compétence du conseil des prud'hommes. Donc j'aurais très bien pu, et personne, je dis bien personne, n'aurait pu me le reprocher, ni la salariée ni encore moins ma hiérarchie, dire à cette dame : "Ben écoutez, madame, vous avez le conseil des prud'hommes, vous avez les organisations syndicales, allez-y, moi, j'ai pas de compétence pénale là-dessus, c'est du pur prud'homme." [...] Voilà, là c'était une personne d'origine malgache, qui ne savait pas vraiment écrire, je lui ai fait un mail, ça m'a pris cinq minutes, elle a fait un copier-coller, elle l'a envoyé à son employeur. [...] J'estime que, dans les cas où les salariés sont complètement démunis, n'ont pas de ressources, on se doit, je trouve que ça fait partie du métier, d'aller au-delà des principes de déontologie.»

Les contradictions internes sont donc nombreuses et les consultations organisées par les inspecteurs du travail se rapprochent, dans certaines circonstances et pour certains salariés, des activités de conseil réalisées par les syndicalistes. Mais dans le même temps, le caractère plus institutionnalisé, et en réalité étatique, du rôle contraint doublement les agents de contrôle, à la fois dans le public qu'ils reçoivent et dans les limites qu'ils se fixent.

La focalisation de l'enquête sur le conseil aux salariés permet donc d'insister sur la production du droit du travail « par en bas », via une activité qu'on a qualifiée de proto-juridique dans la mesure où elle est le plus souvent la première étape - et parfois même la seule étape - de « juridicisation » ou de « judiciarisation » d'un sentiment d'injustice issu de difficultés dans l'entreprise. Cette activité n'en est pas moins portée par des spécialistes du droit du travail, acteurs de l'espace du droit du travail axé vers la défense des salariés. Une analyse globale de cet espace ferait d'ailleurs apparaître d'autres acteurs et groupes professionnels, par exemple les avocats. Ceux-ci arrivent néanmoins après les syndicalistes et les contrôleurs du travail, puisqu'ils effectuent moins un travail de filtrage et de pédagogie que de préparation à l'entrée de l'affaire et du justiciable dans l'arène judiciaire. Il s'agit bien alors d'une forme de division du travail entre les différents acteurs de cet espace, qui d'ailleurs, au niveau local, se connaissent, se rencontrent parfois, échangent des informations. 
Mais on a pu voir tout au long de l'article que syndicalistes et inspecteurs du travail n'exercent pas de la même manière leur activité de conseil. Il faut donc aller au-delà de la convergence apparente du rôle pour en saisir les principes de différence. Le premier tient dans la diversité des contextes juridiques et organisationnels. Alors que les syndicalistes sont globalement libres de donner des conseils, n'ont pas véritablement de comptes à rendre ni d'autre objectif que de représenter leurs adhérents, voire de produire de la syndicalisation, la situation est très différente pour les agents de contrôle. Ceux-ci sont pris dans des contraintes à la fois déontologiques et administratives qui structurent fortement le rôle et obligent ceux qui défendent une vision politique (c'est-àdire ici favorable aux salariés) à jouer avec ces règles et ces contraintes. Une deuxième différence tient alors à l'ethos de ces groupes, aux dispositions qui les portent à jouer ce rôle. Pour les organisations syndicales, et même si certaines d'entre elles sont souvent réticentes à jouer le jeu du droit, le conseil juridique aux salariés est partie prenante du répertoire d'action syndicale, et le droit qui est travaillé est immédiatement politisé ${ }^{41}$. Du côté des agents de contrôle, l'enjeu est fort différent, et l'on retrouve chez eux les formes classiques d'exercice plus ou moins engagé d'une activité professionnelle, avec les différences internes que l'on y retrouve habituellement selon les dispositions des acteurs à exercer des activités à la fois professionnelles et militantes. Enfin, la troisième différence renvoie aux usagers, ici aux salariés venus demander des conseils. Même si l'article ouvre à de nouvelles recherches directement centrées sur eux, on voit la différence de rapport au droit et à l'institution dans la manière de venir vers les uns ou les autres. Les agents de contrôle étant pris dans un cadre étatique et bureaucratique, ce sont donc logiquement des individus déjà préparés à la rencontre des institutions qui viennent chercher conseil à la direction départementale du travail. Au contraire, et sauf quand les uns guident les salariés vers les autres (par exemple quand les contrôleurs du travail suggèrent d'aller vers le syndicat car ils ne se sentent pas autorisés à aller au-delà de leur rôle de renseignement), les consultations syndicales apparaissent comme les premiers interlocuteurs des salariés les plus démunis, venus des PME ou des TPE et donc dépourvus de représentants du personnel dans leur entreprise, ayant entendu parler de ces consultations syndicales mais extrêmement éloignés des contraintes et des ressources du droit. C'est dire que ces espaces de consultation permettent aux organisations syndicales de retrouver une partie de leur rôle premier, qui est d'« équiper » les salariés les moins organisés de ressources collectives leur permettant de défendre leurs intérêts face à l'employeur.

41. Même si le respect du droit est une exigence, aussi bien produite par la nécessité d'être pris au sérieux que par l'existence d'une disposition juridique chez les syndicalistes chargés du conseil, on voit que l'activité est moins soumise à la nécessité de la double conformité juridique et syndicale que par exemple chez les conseillers prud'hommes. 
Laurent Willemez est professeur de sociologie à I'UVSQ, directeur du laboratoire PRINTEMPS (CNRS-UVSQ). Ses recherches portent notamment sur la sociologie du droit du travail, au croisement de la sociologie de l'engagement, de la sociologie du travail et de la sociologie du droit et de la justice. II a co-dirigé avec Hélène Michel des recherches sur les conseils de prud'hommes. II publiera en 2017 Le travail dans son droit. Sociologie historique du droit du travail (1892-2017), Paris, LGDJ.

laurent.willemez@uvsq.fr 